\title{
Models of well-orderings
}

\section{Elizabeth Alice Wachs}

Formal theories of structures of the form $(\alpha, \leq, \vec{f})$, where $\alpha$ is an ordinal and $\vec{f}$ is a collection of arithmetic operations on $\alpha$, have been studied by Mostowski and Tarski [4, 5], Ehrenfeucht [2], Feferman [3], and Doner [1]. In Part $I$ of this thesis we examine the order types of nonwell-ordered (non-standard) models of the first order theories of such structures and in Part II we answer some questions which arise out of the methods used in the earlier part. However, in a sense the bulk of this thesis is just a study of the relation of congruence modulo $\omega^{n}$ on the class of ordinals.

We use the "back-and-forth" criterion for elementary equivalence introduced by Fraïssé and independently by Ehrenfeucht. Often there is an "algebraic" equivalent to the back-and-forth criterion and it is in this role that the papers cited place the relation of congruence modulo $\omega^{\omega}$ on ordinals.

A study of the models of the first order theories of these ordinal arithmetics must involve a study of various monadic weak second order theories by virtue of the mutual interpretability of the monadic weak second order theory of $(\alpha, \leq)$ and the first order theory of $\left(\omega^{\alpha}, \leq,+\right)$. Moreover, ordinals $\alpha$ and $\beta$ are elementarily equivalent as ordered structures if and only if they are equivalent with respect to the corresponding monadic weak second order language, and this occurs if and only if, for all $n \in \omega, \alpha$ is congruent to $B$ modulo $\omega^{n}$.

We consider a class of order types which contains the ordinals, and in this class elementary equivalence and monadic weak second order equivalence

Received 27 October 1976. Thesis submitted to Monash University, May 1976. Degree approved, October 1976. Supervisor: Professor J.N. Crossley. 
do not coincide. Accordingly we describe two extensions to this class of the relation of congruence modulo $\omega^{n}$. From this we obtain a description of the order types of all models of the first order theory

$$
T=n\{T h(\alpha, \leq): \alpha \text { an ordinal }\} \text {. }
$$

Separately we analyse the operations of ordinal addition and multiplication in the context of this class of order types. The analysis is in terms of an approximation to the Cantor normal form representation of ordinals. Once this is done we complete our argument by a fairly routine modification of the classical proofs. In this way we extend Ehrenfeucht's results to the corresponding classes of non-standard models. This takes us to the limit of what can be achieved by an analysis based on an approximation to Cantor normal form. We briefly consider possible extensions of these results to non-standard models of other ordinal systems.

In the first two sections of Part II we are concerned with a connection between algebraic equivalents of the back-and-forth criterion for elementary equivalence of models in a class $M$ and the form of the Lindenbaum algebra of the first-order theory of $M$. We relate our work in Part I on the theory $T$ to more general results of Hanf. In particular we describe an unordered basis for the Lindenbaum algebra of $T$ and further we show that this algebra has an ordered basis of order type $\omega^{\omega}(1+\eta)$.

A number of our results in Part I concerning elementary equivalence may alternatively be deduced from a preservation theorem of Feferman and Vaught. This theorem applies to a large class of "product" structures and relates the first order theory of the product structure to the second order (sometimes weak second order) theory of its index structure. In our last section we look at certain product structures which are defined by reference to the first order theory of their index structures and obtain associated preservation results not deducible from the work of Feferman and Vaught.

\section{References}

[1] John Doner, Definability in the extended arithmetic of ordinal numbers (Dissertationes Mathem,ticae (Rozprawy Matematyczne), 96. Pan̂stwowe Wydawnictwo Naukowe, Warszawa, 1972). 
[2] A. Ehrenfeucht, "An application of games to the completeness problem for formalized theories", Fund. Math. 49 (1960-1961), 129-141.

[3] Solomon Feferman, "Infinitary properties, local functors, and systems of ordinal functions", Conference in Mathematical Logic - London 170, 63-97 (Lecture Notes in Mathematics, 255. Springer-Verlag, Berlin, Heidelberg, New York, 1972).

[4] Andrzej Mostowski and Alfred Tarski, "Arithmetical classes and types of well ordered systems", Bulz. Amer. Math. Soc. 55, Abstract $78 \mathrm{t}(1949), 65$.

[5] Andrzej Mostowski and Alfred Tarski, "Arithmetical classes and types of well ordered systems: Errata", Bulz. Amer. Math. Soc. 55 (1949), 1192 . 